\title{
news
}

\section{news news news news news news news news}

\section{Mental health research challenges in Afric a}

An increasing number of epidemiological mental health studies have been carried out in sub-Saharan Africa in the last decade. However, in developing short-, medium- and long-term strategies, it is clear that further research will have to be carried out in order to provide the evidence necessary to strengthen the mental health care systems of subSaharan Africa, say Florence K Baingana, World Bank senior health specialist, Atalay Alem, psychiatrist at Amanuel Psychiatric Hospital, Addis Ababa University, Ethiopia, and Rachel Jenkins, director of the WHO UK Collaborating Centre, Institute of Psychiatry, Kings College London, writing in the new World Bank publication Disease and Mortality in Sub-Saharan Africa.

Some of the recommended research areas that could be pursued are:

- Cross-sectional and longitudinal studies of mental disorders, including validation of the standardised testing instruments, to establish the epidemiology and causative and risk factors, as well as links to sexual abuse, violence against women, HIV/AIDS and conflicts.
- Multisite studies on the mental well-being of children to establish the incidence and prevalence of mental disorders and links to abuse, malnutrition, conflicts, poverty and vulnerability.

- Stigma and other cultural beliefs.

- Cost-effectiveness, cost-minimisation, and cost-benefit analyses should be undertaken to provide government officials and funding agencies the necessary economic perspective.

- Family, twin, and adoption studies in Western countries have provided evidence of the genetic contribution to the aetiology of depressive disorders, as well as the role of the non-shared environment in the causation of depression. Thus, examination of gene-environment interactions is essential.

- Defective neurotransmission and neuroendocrine receptor responses are associated with depression. It is still unclear whether associations with neurological function represent cause or effect in the pathogenesis of depression and whether neurological function is the major risk factor for depression in sub-Saharan Africa.

- Cost-effectiveness of cognitive behavioural therapy in primary health care settings. 
- Evaluations of mental health services in sub-Saharan Africa.

Increasing the policy and service development and the clinical and research professional capacity in sub-Saharan African countries and stemming the flow of skilled health professionals to wealthy countries are key to developing sustainable, locally appropriate programmes.

Source: www.worldbank.org

\section{Intemational Conference on} Philosophy, Psychiatry \& Psychology

The 1Oth International Conference on Philosophy, Psychiatry \& Psychology is to be held at Sun City from 26 to 30 August 2007, hosted by the International Network of Philosophy \& Psychiatry (INPP) and the South African Society of Psychiatrists (SASOP). The conference is endorsed by the World Psychiatric Association (WPA), the African Association of Psychiatrists and Allied Professions (AAPAP), the Psychology Society of South Africa (PsySSA), and the Philosophical Society of Southern Africa (PSSA). The Annual General Meeting of SASOP will also take place at the conference.

The theme of the conference is 'Hypotheses, Neuroscience \& Real Persons', and the following preliminary categories have been identified:
- conceptual advances and challenges in diagnosis and treatment

- theoretical advances and challenges in the neurosciences/ psychopharmacology/psychophysiology

- diagnostic classification systems: the future

- scientific challenges at the edge in psychiatry/psychology

- ethics in mental health theory and practice

- relationships and interpersonal patterns - advances and challenges

- language and troubled minds and brains

- psychotherapy - conceptual issues of practical relevance

- cognitive neuroscience/cognitive psychology

- phenomenology of experiences in distress and difficulty

- analysis and interpretation in diagnosis/treatment

- computer modelling and Cyborg

- subjectivity in psychopathology: 'What is it like ...?'

- reality: world views and psychosis

- causation of mental disorder.

The deadline for abstracts is 31 March 2007 and further submissions are welcomed. For more information visit the website, or contact Sonja du Plessis at Londocor, sonja@londocor.co.za

Source: www.ppp2007.co.za 